\title{
Model construction of actuation performance of a photo-bending crystal using machine learning-based regression.
}

\author{
Kazuki Ishizaki, ${ }^{1}$ Yuki Hagiwara, ${ }^{1}$ Hideko Koshima, ${ }^{2}$ Takuya Taniguchi, ${ }^{3}$ Toru Asahi ${ }^{1,2}$ \\ ${ }^{1}$ Graduate School of Advanced Science and Engineering, Waseda University, Tokyo, Japan, \\ ${ }^{2}$ Research Organization for Nano \& Life Innovation, Waseda University, Tokyo, Japan, \\ ${ }^{3}$ Center for Data Science, Waseda University, Tokyo, Japan \\ kazuki.143@toki.waseda.jp
}

Acrtuator materials convert input energy into mechanical motion. In recent years, organic actuator materials have attracted attention from the expectations of applications such as soft robots and flexible electronics. Among such actuation materials, photomechanical crystals are expected as a novel type of actuators because these crystals convert light energy into mechanical work due to photoisomerization of photochromic molecules. The actuation properties of photomechanical crystals should be characterized. However, deflection and force, which are crucial for actuators, are dependent on experimental conditions such as light intensity and crystal size, and thus the number of combinations under different conditions is infinite. This causes difficulty in obtaining the relationship between the experimental conditions and actuation outputs. To solve this problem with photo-bending crystals, we applied a machine learning-based regression approach, and then constructed response surfaces of deflection and force.

The deflection and force of the photo-bending crystal were analyzed in the following steps: (1) experimental observations/measurements of deflection and force, (2) feature extraction by exponential fitting, and (3) polynomial regression and variable selection (Fig. 1). In the first step, the deflection of the photo-bending crystal was observed by using a microscope, and the force was measured as the blocking force of the photo-bending (Fig. 1a). The deflection and force behaviors of the photo-bending crystal were measured by changing the light intensities and crystal sizes. In the second step, both deflection and force were fitted to exponential equations for bending and unbending processes and extracting features of the maximum value and time constants at all situations (Fig. 1b). In the third step, the obtaining features are analyzed using polynomial regression, variable selection, and analysis of variance to determine the parameters that influence deflection and force. Through this process, the response surfaces of deflection and force of a photo-bending crystal are statistically constructed by machine learning regression (Fig. 1c), and obtained models were interpreted by chemical and material mechanics. We have found that this machine learning-based regression is useful for relating experimental conditions and actuation outputs, and thus, can be used to control and optimize other functions of stimuli-responsive crystals. This research work was published as an article [1].

(a)
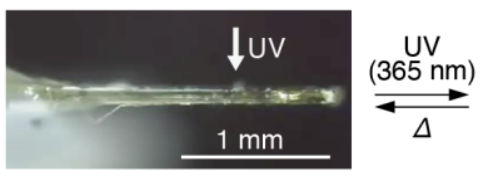

(b)

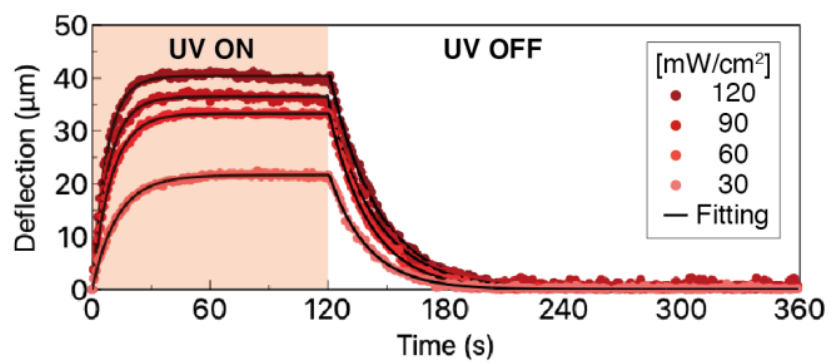

(c)

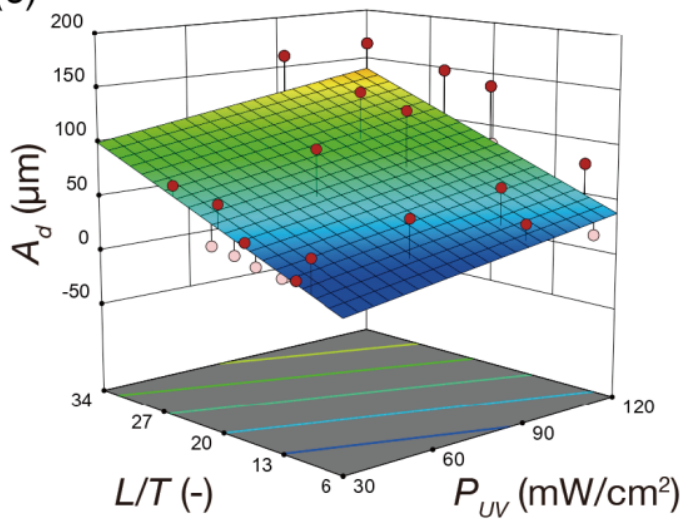

Figure 1. (a) Observation of bending motion, (b) The time course of the deflection and (c) Three-dimensional response surfaces.

[1] K. Ishizaki, R. Sugimoto, Y. Hagiwara, H. Koshima, T. Taniguchi, T. Asahi, CrystEngComm, 2021, DOI: 10.1039/d1ce00208b.

Keywords: Regression analysis; Modeling; Photomechanical bending; Blocking force; Photoisomerization 